A N N A L E S Annales de Bretagne et des Pays de l'Ouest

Anjou. Maine. Poitou-Charente. Touraine

114-4 | 2007

Varia

\title{
Saint-Dimitri, un cimetière orthodoxe de Beyrouth
}

\section{May Davie}

\section{OpenEdition}

\section{Journals}

Édition électronique

URL : http://journals.openedition.org/abpo/457

DOI : $10.4000 / a b p o .457$

ISBN : 978-2-7535-1508-6

ISSN : 2108-6443

Éditeur

Presses universitaires de Rennes

Édition imprimée

Date de publication : 30 décembre 2007

Pagination : $29-42$

ISBN : 978-2-7535-0598-8

ISSN : 0399-0826

Référence électronique

May Davie, «Saint-Dimitri, un cimetière orthodoxe de Beyrouth». Annales de Bretagne et des Pays de I'Ouest [En ligne], 114-4 | 2007, mis en ligne le 30 décembre 2009, consulté le 30 avril 2019. URL: http://journals.openedition.org/abpo/457 ; DOI : 10.4000/abpo.457 


\title{
Saint-Dimitri, un cimetière orthodoxe de Beyrouth
}

\author{
May DAVIE \\ Professeur à l'université de Balamand (Liban) \\ CEHVI - université de Tours
}

L'idée que l'on a habituellement des cimetières dans le monde arabe s'est construite à partir des cimetières musulmans, avec leurs paysages classiques composés d'alignements de stèles de facture simple et quasi identique $^{1}$, sans monuments funéraires ou tout autre signe ostentatoire. En Islam, tous les croyants sont égaux devant le Très-Haut. Le signalement, dans la mort, d'une différence sociale ou ethnique est proscrit par la religion et du reste très mal vu par la société.

Dans le monde arabe, on trouve pourtant de nombreux cimetières chrétiens. Au Maghreb, ils sont structurés selon une logique propre, s'agissant généralement de cimetières de militaires occidentaux ou d'anciens colons, et sont à présent et pour la plupart désaffectés. Au Machreq, les cimetières chrétiens appartiennent à des minorités autochtones ralliées aux différents rameaux du christianisme oriental et sont toujours en usage. Comparés aux cimetières musulmans, ils ont une tout autre allure. L'exemple traité ici, Saint-Dimitri, est le cimetière de la communauté orthodoxe de Beyrouth, une des plus anciennes de la cité, ses racines remontant à la naissance du christianisme dans cette région du monde.

Saint-Dimitri est singulier dans son genre et pour son époque, par l'originalité de son architecture, l'exubérance de ses décors et le site antique mythique qu'il occupe. Fondé au début du XIx siècle, lors de l'occupation égyptienne (1832-1840), il se développa durant la restauration ottomane. Cette période transitionnelle éclaire autant la société beyrouthine pré-moderne qui résidait dans la ville intra muros, que l'évolution de sa structure sociale et de ses coutumes, au temps de l'avènement du capitalisme marchand et de la modernité institutionnelle et urbanistique des réformes ottomanes (les tanzimat), impulsées

1. Deux stèles sont habituellement dressées sur la tombe, l'une à la tête et l'autre au pied du corps en terre. 
durant la même époque. Ce cimetière reflète encore l'homogénéisation sociale qui a touché la communauté depuis l'Indépendance, notamment entre 1950 et 1975. Aujourd'hui rattrapé par l'urbanisation et lui-même très dense et dégradé, il nous renvoie, au surplus, l'image de cette ville désordonnée qu'est devenue Beyrouth depuis plus d'un quart de siècle. Ce sont ces ruptures et leurs correspondances dans le cimetière même que nous décrirons dans ce texte.

\section{Les orthodoxes de Beyrouth}

Les orthodoxes de Beyrouth sont des chrétiens de langue et culture arabes et de rite byzantin. Ils relèvent du patriarcat d'Antioche dont l'autorité s'étend sur le territoire de ce qu'on appelle la Grande Syrie historique ${ }^{2}$. Si certains parmi eux affirment être originaires de la péninsule arabique, la plus grande partie vient des villes et des bourgs de Syrie, d'Asie Mineure, de la Grèce et de ses îles. Ces populations, parlant plusieurs langues, ont toutes été arabisées par le poids de l'histoire. Elles se sont encore mêlées à des Russes blancs, des Ukrainiens et des Géorgiens, arrivés à Beyrouth depuis la Révolution d'octobre. Durant le Mandat français (1918-1943), elles furent rejointes par d'autres réfugiés originaires du sandjaq d'Alexandrette ${ }^{3}$. Depuis, l'exode vers Beyrouth a touché des Palestiniens et surtout la population du mont Liban. C'est dire que les orthodoxes de Beyrouth sont le fruit de brassages ethniques et culturels complexes.

En arabe populaire, les orthodoxes ont toujours été appelés roum (romains), en référence à l'empire romain d'Orient et à l'Église officielle dont ils sont, localement, les héritiers spirituels. C'est ce nom que les Arabes musulmans donnaient, après leur conquête de la Syrie, aux Byzantins comme à ceux de leurs compatriotes qui professaient la même foi que Constantinople, la nouvelle Rome.

Avant le XVIII siècle, on les retrouve encore sous l'appellation « melchites " (royalistes), car ils étaient restés fidèles aux enseignements de l'Église primitive lors des schismes qui l'avaient secouée à plusieurs reprises. L'empire ottoman et, à sa suite, l'État libanais les reconnaissent comme roum orthodox. Cette expression signifie littéralement "romains orthodoxes ". Mais elle a été traduite par " grecs orthodoxes ", en raison du rite byzantin qu'ils partageaient avec les chrétiens de Constantinople et de Grèce. Ce sont les missionnaires et les consuls occidentaux qui leur ont tout d'abord accordé l'appellation "Grecs ", dans leurs écrits des XVIII et XIX ${ }^{\mathrm{e}}$ siècles, cherchant, de toute évidence, à réserver la dénomination " romains "

2. Qui correspond approximativement à la province ecclésiastique romaine Oriens (l'Orient), qui allait du Taurus jusqu'à la péninsule sinaïque et incluant la Mésopotamie (DAVIE, May, Atlas historique des grecs-orthodoxes de Beyrouth et du Mont-Liban 1800-1940, Beyrouth, Dar an-Nahar, 1999)

3. Province syrienne cédée par la France à la Turquie en 1939, en contrepartie de sa neutralité durant la Seconde Guerre mondiale qui s'annonçait. 
aux seuls fidèles du siège pontifical du Vatican, dans la "vraie" Rome 4 . Désirant enfin écarter toute confusion au plan ethnique, les orthodoxes ont eux-mêmes récemment adopté l'appellation " orthodoxes d'Antioche ", à consonance ecclésiale unique ${ }^{5}$.

Saint-Dimitri est l'exacte réplique de ce visage cosmopolite des orthodoxes de Beyrouth. Le paysage monumental de ce cimetière, ses symboles et ses décors marquent les modifications des rapports de pouvoir au sein de la communauté durant le XIX ${ }^{\mathrm{e}}$ siècle et l'évolution ultérieure.

\section{Saint-Dimitri}

Saint-Dimitri est situé sur un replat au pied d'une falaise abrupte, au cœur des quartiers résidentiels orientaux de Beyrouth. Le cimetière a la forme d'un triangle allongé, entourant une église construite sur une partie légèrement surélevée de ce replat et précédée par de larges escaliers et par une allée monumentale menant vers l'extérieur. Le bâtiment de l'église fut inauguré en 1900, mais le site est beaucoup plus ancien. À l'arrière, vers l'est, on voit l'abside de la chapelle qui a vraisemblablement précédé le sanctuaire actuel. Elle-même serait construite sur un site antique, sans doute un temple païen associé à un point d'eau. Au XIX ${ }^{e}$ siècle, et plus récem-

Figure 1 - L'entrée monumentale du sanctuaire (cliché de l'auteur)

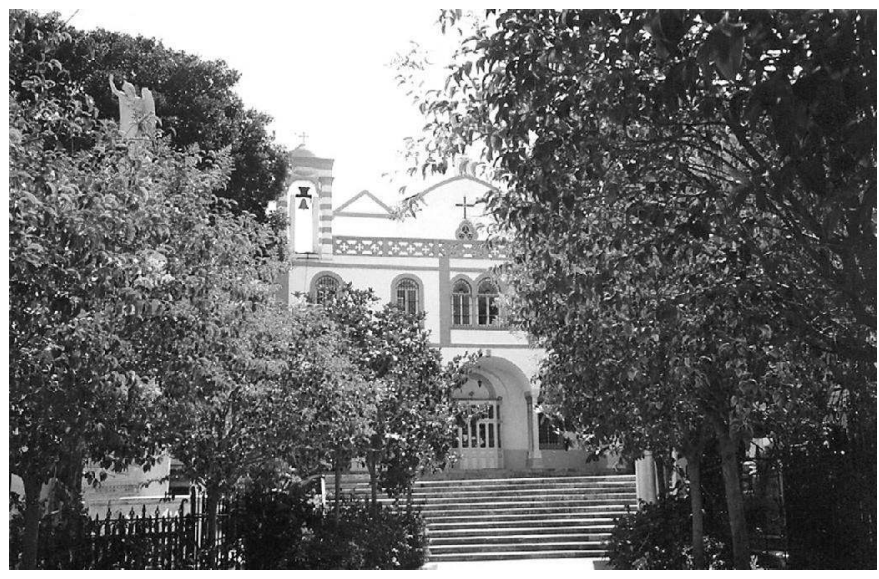

4. Ces derniers les traitaient également de "schismatiques ", à l'instar de toute Église orientale non soumise à l'autorité du pape.

5. Des auteurs contemporains (HEYBERGER, Bernard [dir.], Chrétiens du Monde arabe, un archipel en terre d'islam, Paris, Éditions Autrement, coll. " Mémoires ", n 94, 2003, p. 811) continuent à livrer une catégorisation sans nuances des chrétiens d'Orient, plaçant toujours les orthodoxes arabes du patriarcat d'Antioche dans la catégorie des "Grecs " et entretenant la confusion entre des réalités différentes. 
ment durant les années 1990, des sarcophages antiques ont été trouvés autour de ce site, suggérant la présence de propriétés suburbaines, avec villas et tombeaux, appartenant aux milieux aristocratiques de Berytus, la colonie romaine qu'était alors Beyrouth ${ }^{6}$.

Saint-Dimitri se dit Mar Mitr en arabe : Mar vient du syriaque et signifie saint; Mitr est l'arabe populaire de Dimitri. Mais le nom officiel du site est Qiddis Dimitrious, qiddis signifiant saint en arabe classique, Dimitrious étant le nom grec arabisé de Dimitri.

Figure 2 - Le plan du cimetière

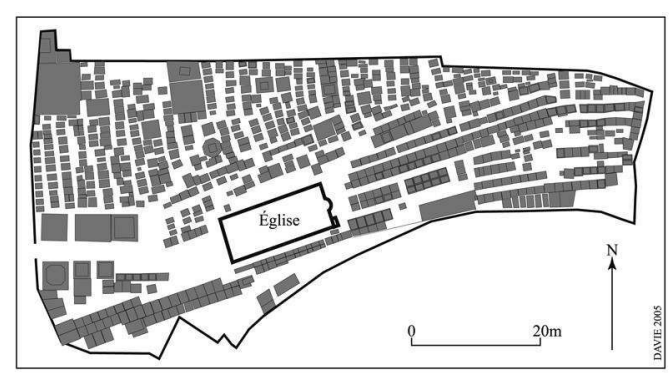

À l'origine, Saint-Dimitri n'était pas une chapelle funéraire, mais un lieu de dévotion, implanté à proximité d'une pinède et dédié à ce saint militaire. D'autres chapelles de ce genre (que l'on désigne localement par deir ou mazar) existaient à Beyrouth, notamment Saint-Élie et Saint-Georges, respectivement au sud, près des dunes de sable, et à l'est, à proximité de l'embouchure du fleuve de Beyrouth. Elles balisaient, depuis l'Antiquité, la limite du territoire urbain, défenses symboliques des espaces verts par lesquels on accédait à l'agglomération. Ces trois saints intercesseurs entre les hommes et leur Créateur étaient particulièrement vénérés par la population locale. Ils étaient en tout cas réputés exauceurs, pour être aussi visités et par les musulmans de Beyrouth et par des voyageurs occidentaux qui en ont laissé des descriptions ${ }^{7}$.

Saint-Dimitri prit une fonction cémétériale durant la première moitié du XIX ${ }^{\mathrm{e}}$ siècle, en conséquence d'une mesure égyptienne d'urbanisme et d'hygiène ordonnant le déplacement des cimetières hors les murs. Auparavant, le cimetière orthodoxe jouxtait la cathédrale Saint-Georges, dans la ville intra muros, aujourd'hui dans le centre de la ville moderne. Depuis cette date, trois cimetières orthodoxes furent construits dans les banlieues vouées à l'agriculture et pratiquement vides d'habitations, en périphérie

6. Voir JIDEJIAN, N., Beirut through the Ages, Beirut, Dar el-Mashreq, 1973.

7. Notamment LAMARTINe, Alphonse de, Souvenirs, impressions, pensées et paysages pendant un voyage en Orient, 1832-1833, Paris, Hosselin, 2 vol., 1835, p 317; CARNE, J., Syria, the Holy Land, Asia Minor, Londres, Fisher Son \& Co, 3 vol., p. 9-10; et Blondel, E., Deux ans en Syrie et en Palestine, Paris, 1840, p. 66. 
des faubourgs naissants. Ces zones sont toutes aujourd'hui envahies par l'urbanisation dense. Parmi ces trois, Saint-Dimitri reste le plus important ${ }^{8}$. Il concerne plus de la moitié des orthodoxes de Beyrouth, tous ceux qui ont choisi de s'installer à l'est de l'agglomération lors de l'expansion hors muraille, vers la fin du XIX ${ }^{\mathrm{e}}$ siècle. C'est surtout le cimetière des familles patriciennes, celles de l'aristocratie urbaine et de la haute bourgeoisie qui ont dirigé la communauté et gouverné la cité durant le XIXe siècle et le début du $x x^{e}$ siècle, au sein des Conseils ottomans locaux. Le mode d'implantation des sépultures et l'organisation générale du cimetière dévoilent justement cette hiérarchie.

\section{La fondation}

Un inventaire des tombes, de leurs formes, de leurs décors et de leur disposition, a permis de diviser schématiquement le cimetière en trois parties et trois périodes historiques correspondantes.

Le côté nord, en contrebas de l'église et de l'allée monumentale, semble matérialiser l'installation première, la fondation. L'architecture et l'épigraphie donnent à penser que cette période inaugurale va de 1835, date estimée du transfert de Saint-Georges, jusqu'à la fin des années 1860. En raison des embellissements constants et des besoins d'une population en expansion, des reconstructions ou des remaniements ont fait disparâ̂tre nombre de sépultures antérieures. Mais plusieurs rangées de tombes anciennes subsistent encore dans leur état original. Elles sont austères d'aspect et semblables de forme et de taille, des structures rectangulaires de couleur terne et hautes d'environ un demi-mètre, en fait des caveaux en pierre de grès, à moitié enfouis dans le sol et couverts de terre battue ou d'une simple dalle de pierre taillée. Aucun ouvrage particulier, stèle ou croix, ne surmonte ces vieilles sépultures. Aucun ornement ne les enjolive non plus.

Pour être contrainte par un même modèle et arrangée selon un schéma régulier apparemment préétabli, cette installation n'est pas pour autant aléatoire. Un partage réfléchi de l'espace mortuaire est décelable. Les tombes des vieilles lignées de notables urbains ${ }^{9}$, comme la famille Trad, occupent le côté nord-ouest en bordure immédiate de la rue d'où elles sont bien en vue. À l'angle, deux d'entre elles furent remaniées pour porter des monuments imposants. L'autre lieu privilégié par les anciennes familles beyrouthines est l'abord du sanctuaire, en dessous ou en contrebas de la

8. Les deux autres sont Mar Élias Btina (Saint-Élie) et Saydet an-Niyah (Notre-Dame de la Dormition).

9. Sur ces familles, les mythes d'ancienneté et la notabilité à Beyrouth durant le XIX ${ }^{\mathrm{e}}$ siècle, voir : DAVIE, May, "Les familles orthodoxes à travers les cahiers du Badal 'Askariyyet ", Annales d'Histoire et d'Archéologie de l'Université Saint-Joseph, Beyrouth, vol. 5, 1986, p 144 et DAVIE, May, , "Villes, notables et Pouvoir : les Orthodoxes de Beyrouth au XIX siècle ", dans Bourgeoisies et notables dans le Monde Arabe, XIX et XX siècles, Les Cahiers de la Méditerranée, CEMO, n 45, 1992, Nice, p. 183-202. 
Figure 3-Une tombe à l'ancienne (cliché de l'auteur)

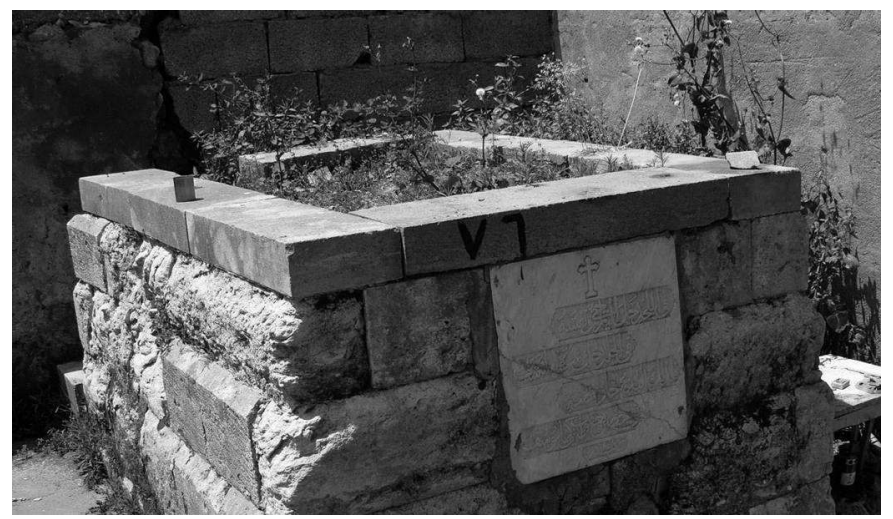

galerie qui court sur son flanc nord. Quant aux minorités russes, grecques et chypriotes, on les trouve pour la plupart concentrées dans la partie centrale, non loin du mur d'enclos septentrional.

Nombre de ces tombes anciennes sont anonymes. Mais pour la plupart, elles portent une plaque de pierre posée ou encastrée sur le côté et gravée d'une inscription de belle facture. L'intérêt de ces premières sépultures réside justement dans la graphie de ces textes, les beaux caractères en arabe naskhi, thulthi ou reyhani ${ }^{10}$ se détachant en relief sur un fond excisé et généralement délimité par un listel. Les plus anciennes ne signalent que

\section{Figure 4 -Symboles chrétiens sur une épitaphe de 1840 \\ (cliché de l'auteur)}

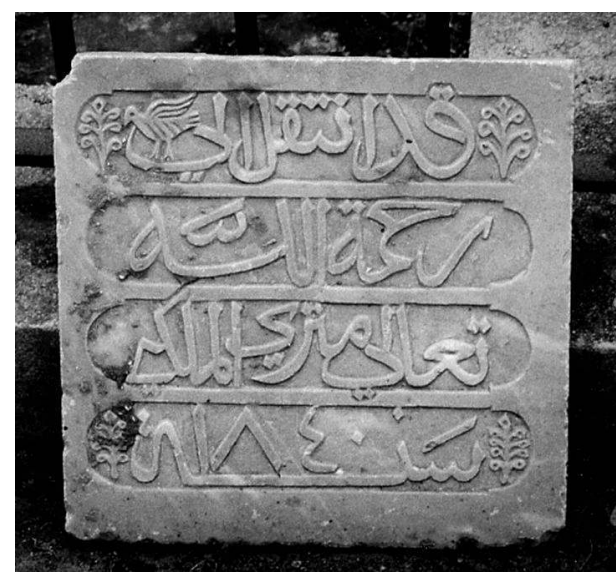

10. Types de calligraphie arabe et ottomane. le nom du titulaire ainsi que la date de son décès et ne comportent pas d'ornements. Certaines plaques sont certes gravées d'une colombe, d'une croix byzantine ou d'un arbre de vie, mais le dessin est naif et discret, suggérant une indifférence au marquage par des signes chrétiens visibles. N'étaient la présence de ces tombes à Saint-Dimitri et le prénom chrétien du titulaire (Georges, Michel, Constantin, Nicolas...), il serait impossible de les distinguer comme spécifiquement chrétiennes.

Les épitaphes plus récentes sont en revanche longues 
de plusieurs lignes et traitent de thèmes sur l'espérance, la foi en la Résurrection ou la valeur morale de la personne décédée. Elles sont aussi plus ornementées, les lignes de l'inscription étant gravées dans des cartouches excisés et décorés d'un rinceau, d'un cordage ou d'une couronne.

Il se trouve dans ce secteur quelques tombes de Grecs qui ne comportent pas de caveau, l'inhumation s'étant faite directement en terre, une coutume que pratiquaient d'ailleurs anciennement les Arabes chrétiens du Moyen Orient, à l'instar de leurs concitoyens musulmans. L'utilisation du caveau par les orthodoxes daterait-elle de la fondation de SaintDimitri ou s'était-elle simplement généralisée durant cette période? Rien ne permet de trancher ces questions de manière définitive. Durant les fouilles archéologiques effectuées à Saint-Georges durant les années 1990, deux cimetières remontant au Moyen Âge furent exhumés, révélant des mises en terre individuelles, bordées parfois par des blocs de pierres, sans caveau ni cercueil. Du cimetière plus récent qui fut transféré à Saint-Dimitri, ont subsisté peu de vestiges, dont un seul caveau de taille réduite, ne livrant que des renseignements épars qui ne permettent pas de généraliser. L'absence de débris de bois et de clous, et de fragments de croix laisse quand même supposer que caveau et cercueil n'étaient pas alors des pratiques systématiques, comme cela allait être le cas à Saint-Dimitri, un siècle plus tard ${ }^{11}$. En outre, sur les inscriptions retrouvées, seul le nom du défunt est gravé et aucun signe chrétien n'apparaît.

\section{Enjeux et contraintes de l'occupation de nouveaux terrains}

Tout le côté ouest, devant l'église et sur la falaise attenante, fut apparemment conquis entre le dernier quart du XIX ${ }^{\mathrm{e}}$ siècle et la première moitié du $\mathrm{xx}^{\mathrm{e}}$ siècle. Ici, c'est la richesse des sépultures qui saute au regard, s'agissant

11. Les fouilles ont été réalisées sous la direction de Leila Badre. Nous la remercions pour ces renseignements.
Figure 5 - Une épitaphe de 1842,

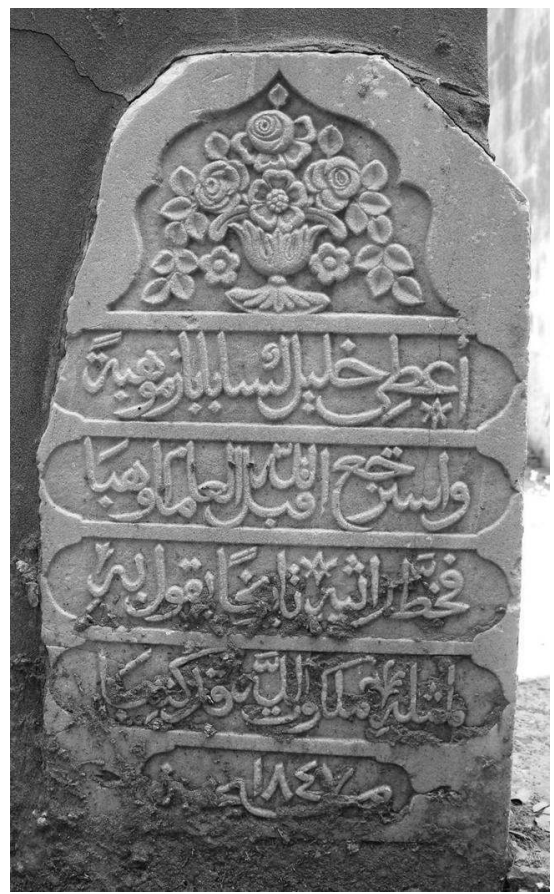


Figure 6 - Une épitaphe calligraphiée tardive (cliché de l'auteur)

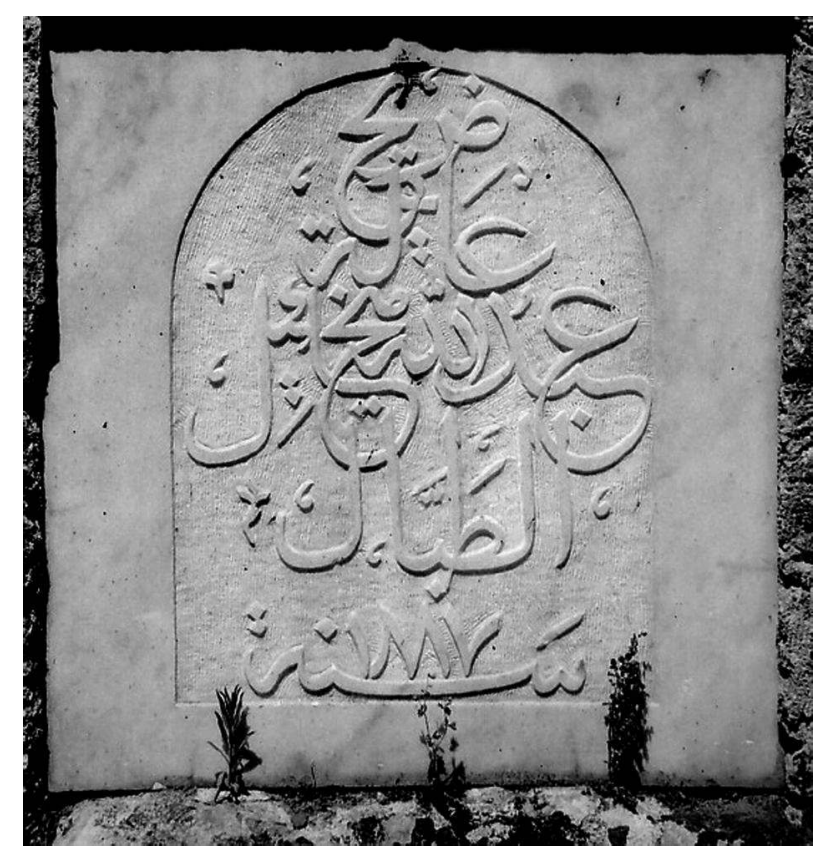

naturellement de la partie du cimetière devenue devanture sociale de l'aristocratie montante et des dynasties marchandes, qui ont su tirer profit de l'arrimage de la ville et de son port à l'économie mondiale, durant le XIX ${ }^{\mathrm{e}}$ siècle ottoman ${ }^{12}$. Leurs patronymes, Sursock, Jbeily, Debbas, Abou Chanab, Bustros, Terzis et d'autres, sont désormais synonymes de prestige social.

Cette installation tranche, par la forme comme par la signification, avec la fondation antérieure. Les sépultures sont, sans exception, couvertes de monuments imposants, devenant de véritables tombeaux. Elles sont au demeurant organisées en deux schémas différents, suggérant deux politiques d'occupation du sol.

Le premier schéma forme un alignement de mausolées qui bordent les côtés du chemin d'entrée menant au sanctuaire, formant une sorte d'allée aux tombeaux. Les parcelles sont quatre fois plus larges et d'un prix environ dix fois plus élevé qu'ailleurs, et les monuments sont construits sur des podiums entourés de végétation. Cette allée est réservée aux différentes branches de la maison aristocratique éminente du Beyrouth de ce temps, les Sursock. Il faut rappeler que cette famille était associée en affaires avec

12. Sur les conditions d'émergence de ces classes sociales, voir FaWAZ, L., Merchants and Migrants in Nineteenth-Century Beirut, Cambridge, Massachussetts, Harvard University Press, 1983. 
l'aristocratie ottomane d'une part, et alliée par le mariage à l'aristocratie génoise et napolitaine de l'autre. Un de ses membres, Youssef (Joseph), était le doyen de la communauté orthodoxe et le proche conseiller du wali (préfet) de Beyrouth, jusqu'à la chute de l'Empire ottoman ${ }^{13}$. Il convient de constater que les palais des Sursock et leurs parcs luxuriants étaient pareillement disposés le long de la rue de l'Archevêché-Orthodoxe, située non loin du cimetière, dans le riche quartier de Rmeil. À SaintDimitri, tout concourt à produire cet effet de vitrine rappelant, dans la mort comme dans la vie, le statut de cette famille et la force de son ancrage dans la cité et dans son économie.
Figure 7 - Décors baroques ottomans (Cliché de l'auteur)

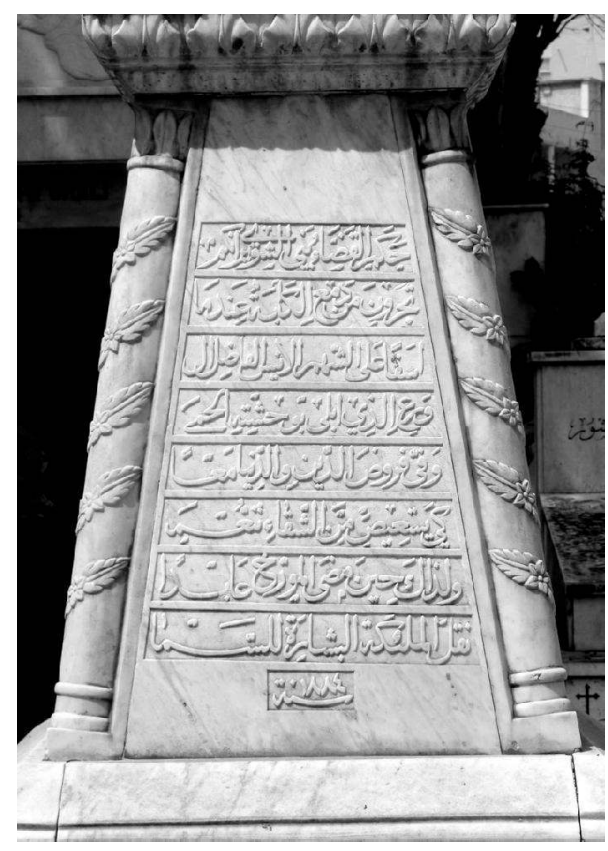

Figure 8 - Tombeaux aristocratiques de l'allée monumentale (cliché de l'auteur)

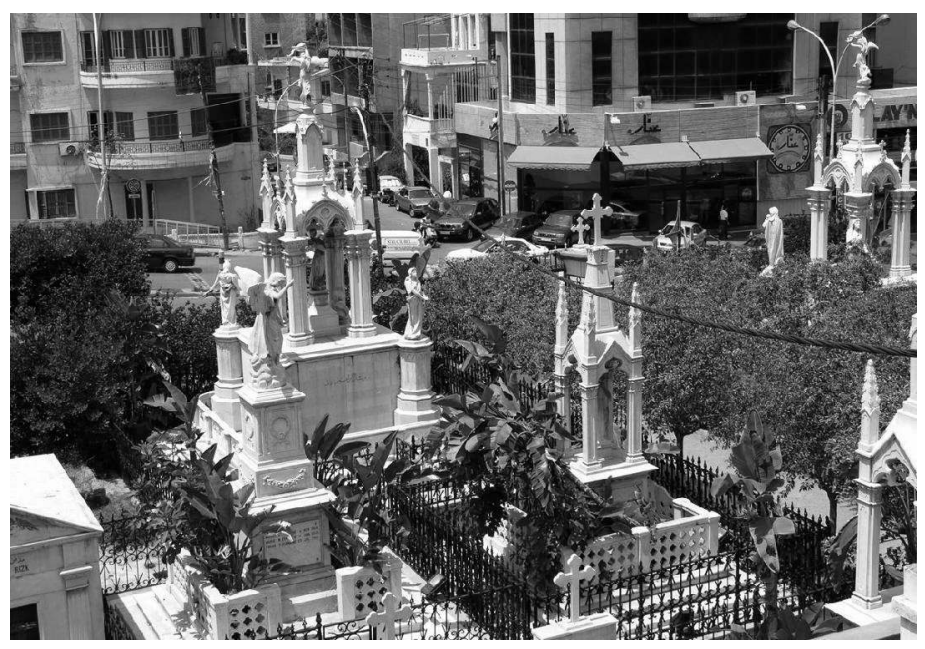

13. Voir ÖzverEn, E., The Making and Unmaking of an Ottoman Port City: Nineteenth-Century Beirut, its Hinterland and the World Economy, Thesis State University of New York, 1990, et Owen R., The Middle East in the World Economy, 1800-1914, London, Methuen,1981. 
Figure 9 - Rangée d'édicules funéraires (cliché de l'auteur)

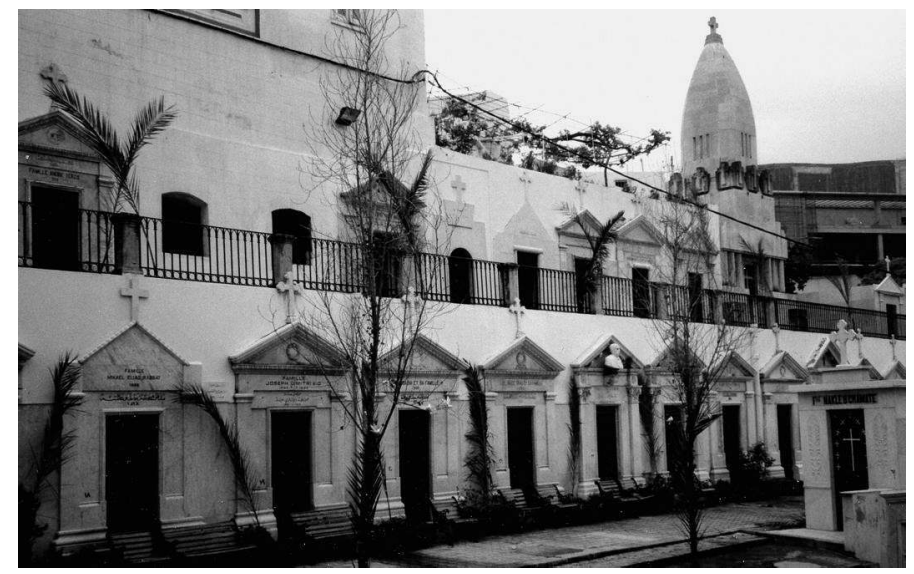

Figure 10 - À l'arrière, une sépulture à baldaquin du $X_{X} X^{e}$ siècle et une reconstruction moderne en béton (Cliché de l'auteur)

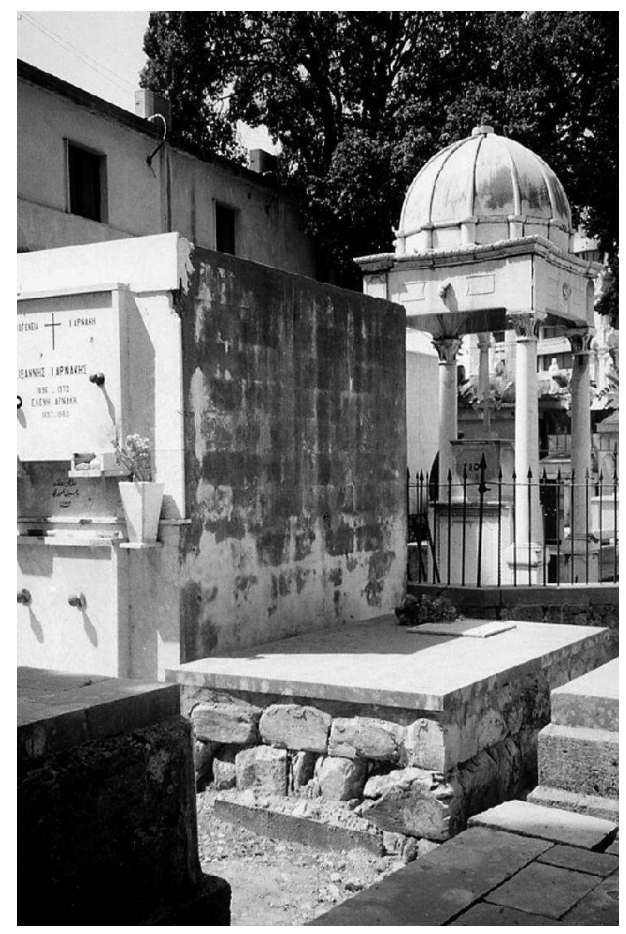

Le ciborium est la figure architecturale privilégiée de cette composition au goût occidental prononcé. Il est décliné dans tous les styles à la mode, le gothique, le classique et le baroque, et utilise des matériaux nouveaux, dont le marbre et le fer, et une statuaire importés d'Italie. L'on relève l'apparition d'une quantité de décors sculptés, surprenants dans le contexte beyrouthin de ce temps (globes, flèches, fleurs, colonnes, pinacles, flammes, livres...), parmi lesquels les signes chrétiens abondent, anges, putti, colombes, et, immanquablement, de grandes croix. Marquer l'espace mortuaire du sceau de la foi chrétienne est maintenant une exigence.

Le second schéma d'implantation consiste en des alignements d'édicules surmontés d'un fronton triangulaire et adossés côte à côte aux escar- 
pements des quatre niveaux qui composent la falaise devant l'église. Tirant profit de la topographie naturelle, ces tombes sont en position panoramique vis-à-vis du sanctuaire et de son cimetière, et aussi du quartier d'où elles peuvent être aperçues. Les surfaces allouées ici sont plus réduites que sur le passage monumental. Pas de place pour le végétal et pas de décor en surcharge. On lit, gravés sur le tympan des portes, les noms des lignées. L'aspect austère que dégagent les rangées de frontons semblables rend toutefois ces tombes tout aussi solennelles que celles sur l'allée aux tombeaux. Comme dans l'allée, la calligraphie arabe ou ottomane n'est plus de mise. Les inscriptions sont en typographie simple, standardisée et parfois même en français. Les invocations disparaissent, cédant la place à la croix byzantine, simple ou tréflée, sculptée dans le marbre et dressée au sommet des mausolées.

Alors que l'espace mortuaire des gens très aisés allait se figer, de nombreuses tombes du côté nord de Saint-Dimitri, celui des familles beyrouthines les plus vieilles, furent remaniées durant cette période. On y ajouta un revêtement en marbre, un monument (cippe, stèle, sarcophage, autel...), des décors baroques et une croix au sommet. Pour être importés d'Occident, ces modèles n'en furent pas moins réinterprétés pour plaire à la demande locale. Certaines tombes, reconstruites dans les années 1960, sont de style moderne et utilisent, copiant toujours l'Europe, des matériaux sombres comme le granite.

\section{À l'image de la modernité beyrouthine}

Le terrain derrière l'église, côté est, commence à se remplir vers 1940. Il se densifie ensuite progressivement, puis de manière rapide, à partir de 1960. Le secteur est caractérisé par des surfaces au sol réduites par rapport aux pratiques antérieures, par l'entassement et le manque de soins, et par l'absence totale de végétation. La morphologie suggère, en tout cas, une expansion en deux temps, une occupation de la surface horizontale juste derrière l'église, suivie par la conquête du côté oriental de la falaise.

L'architecture funéraire moderne qui apparaît à l'arrière de l'église semble contrainte par l'urgence et par le manque apparent de moyens pour des sépultures et des décors soignés. Il s'agit des sépultures de familles dernièrement installées en ville, des cols blancs pour la plupart, qui devaient en outre s'accommoder de l'exiguïté du terrain résiduel de Saint-Dimitri. Les tombes sont pour la plupart d'un géométrisme banal, de simples blocs en parpaings, couverts d'un enduit en ciment peint à la chaux, ou de plaques de marbre quand le défunt ou sa famille en avaient les moyens. L'art funéraire orthodoxe de la phase antérieure s'estompe, plus de statuaire et plus d'objets ou de symboles chrétiens clairs. Le seul moyen d'expression de la foi qui demeure est une petite croix gravée et rehaussée de peinture noire. Mais ce qui reste surprenant est la présence de caveaux superposés sur deux ou trois niveaux, et surtout de cases individuelles, symbole ultime de 
Figure 11 - Entassement et délabrement sur la partie orientale de la falaise (cliché de l'auteur)

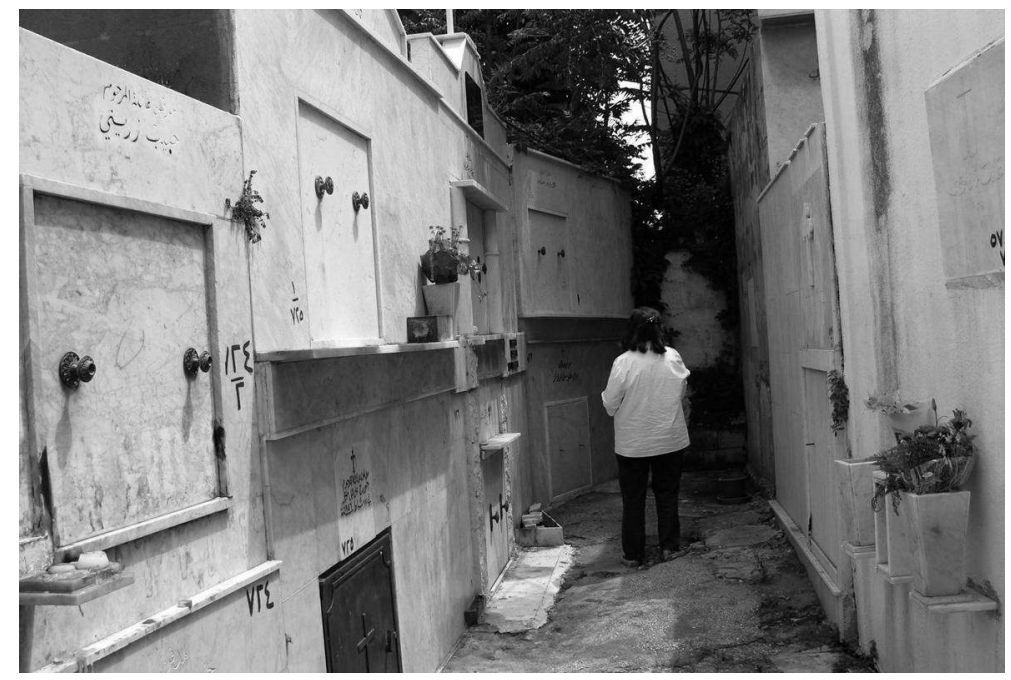

Figure 12 - Remaniements et densification de côté nord (cliché de l'auteur)

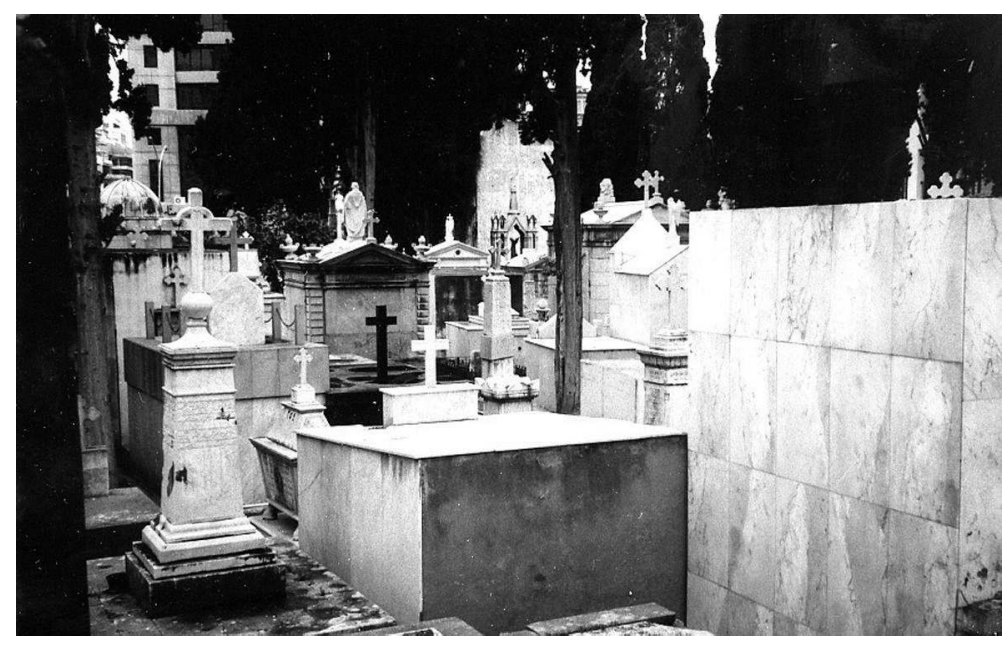

l'anonymat social et de la démocratisation de la mort, par l'industrialisation et la banalisation de la dernière demeure. Contrairement au côté occidental de la falaise où les chambres funéraires s'ouvrent sur l'espace, les assemblages occupent ici les deux bords de la terrasse avec un passage central étroit, une sorte de couloir qui va en se rétrécissant, coincé entre ces étagements sans âme. Durant la saison chaude, la réverbération des rayons 
du soleil sur ce monde minéral transforme les passages en une fournaise répulsive pour la visite et pour tout recueillement. Rien ne rappelle mieux les entassements de logements modestes et les bidonvilles des périphéries pauvres du Beyrouth d'aujourd'hui.

En raison de la croissance démographique existant encore au début des années 1970, puis de la dernière guerre civile (1975-1990), même les espaces morts du cimetière initial, au nord du sanctuaire, ont été occupés par ce genre de structures. Des tombes en béton, sans caractère et de facture pauvre, l'envahissent lui aussi, intercalées entre les anciennes ou bloquant carrément les chemins d'accès. C'est ici, également, que les lotissements des familles éteintes sont récupérés pour les nouveaux venus.

À l'origine installé dans une pinède propice au recueillement et suffisamment grand pour être pratique, Saint-Dimitri fut conçu pour une société orthodoxe certes bourgeoise, mais pour laquelle les signes extérieurs de la richesse n'étaient pas de mise. C'est l'humilité dans la mort qui était plutôt recherchée. Cette société n'éprouvait pas non plus le besoin d'afficher par des signes ostentatoires sa foi chrétienne, évidence tranquille pour elle. Les épitaphes étaient en revanche d'usage courant, pareillement aux pratiques funéraires musulmanes, exprimant le nom du défunt ou des évocations morales ou religieuses.

À partir de 1870, le cimetière s'agrandit et se rénova, pour répondre à de nouvelles exigences démographiques et sociales. Avec la transformation des rapports de pouvoirs, le culte du tombeau, à la mode en Occident durant la même période, rentra à Beyrouth. Apparaissent dès lors, à SaintDimitri, le monument et le décor funéraires et surtout les signes chrétiens les plus ostentatoires, notamment les grandes croix dressées au sommet des tombes et qui se distinguent de loin. Ces éléments de l'iconographie chrétienne occidentale devinrent les fondements de l'art funéraire des orthodoxes. On assiste encore à l'apparition d'inscriptions en langue étrangère à la communauté, notamment le français, une langue devenue de rigueur à Beyrouth où parler une langue occidentale est devenu de bon ton, le signe en tout cas d'un certain statut social.

L'époque contemporaine voit par contre l'encombrement et le délabrement s'installer à Saint-Dimitri. Étouffé par des tombes sans caractère et de mauvaise qualité et par le manque de soins, il perd lentement son cachet et ses repères. Le beau et le symbole importent peu maintenant. Le cimetière se retrouve aussi noyé par l'urbanisation qui l'entoure, devant cohabiter avec les immeubles commerciaux, modernes et disproportionnés, qui ont poussé devant son entrée, depuis l'élargissement de la rue en une voie rapide au trafic automobile incessant, productrice de poussière et bruit.

Notons alors, pour conclure, que les cimetières chrétiens en Orient sont, à leur manière, une clé pour approcher les mutations d'une société. Dans leur organisation transparaît encore la morphologie de la ville dans laquelle ils sont implantés. À Saint-Dimitri, on lit la progressive appropriation du 
cimetière, comme de la ville ${ }^{14}$, par les classes moyennes. Contrairement à l'ancienne bourgeoisie qui exhibait son attachement à la cité par un intérêt manifeste pour son rite et pour les monuments et les signes qui l'expriment, les nouveaux venus sont indifférents à l'aspect comme au sort de leur dernière demeure, dans un groupe au sein duquel ils sont encore anonymes et dans une ville, à leurs yeux, incivile.

\section{RÉSUMÉ}

Saint-Dimitri, est le cimetière de la communauté orthodoxe de Beyrouth. Fondé au début du XIX ${ }^{\mathrm{e}}$ siècle, ce cimetière est singulier par l'originalité de son architecture, l'exubérance de ses décors et le site antique mythique qu'il occupe.

Son histoire renseigne autant sur la société beyrouthine prémoderne qui résidait dans la ville intra muros, que sur l'évolution de sa structure sociale et de ses coutumes, au temps de l'avènement du capitalisme marchand à la fin du $\mathrm{XIX}^{\mathrm{e}}$ siècle. Elle reflète encore l'homogénéisation sociale qui a touché la communauté depuis l'Indépendance, notamment entre 1950 et 1975. Aujourd'hui rattrapé par l'urbanisation et lui-même très dense et dégradé, Saint Dimitri renvoie au surplus l'image de cette ville désordonnée qu'est devenue Beyrouth depuis plus d'un quart de siècle.

\section{ABSTRACT}

Saint Dimitri is the graveyard of the Beiruti Greek Orthodox community. Founded at the beginning of the $19^{\text {th }}$ Century, this cemetery is unique by its original architecture, its exuberant decors and the mythical story of the site it is implemented on.

The examination of the cemetery brightened up the history of the intra muros Beiruti Pre-modern society, its evolution through the $19^{\text {th }}$ Century and its homogenization as from the Independance $20^{\text {th }}$ period.

Today very dense and timeworn, Saint Dimitri looks like the disorganized city of Beirut since 1975.

14. Sur ce mouvement, voir DAVIE, May, Beyrouth et ses faubourgs (1840-1940), une intégration inachevée, Beyrouth, CERMOC, 1996. 